\title{
The impact of common polymorphisms in CETP and $A B C A 1$ genes with the risk of coronary artery disease in Saudi Arabians
}

\author{
Cyril Cyrus $^{1 *}$, Chittibabu Vatte ${ }^{1}$, Awatif Al-Nafie ${ }^{2}$, Shahanas Chathoth ${ }^{1}$, Rudaynah Al-Ali ${ }^{2}$, Abdullah Al-Shehri ${ }^{2}$, \\ Mohammed Shakil Akhtar ${ }^{2}$, Mohammed Almansori ${ }^{2}$, Fahad Al-Muhanna ${ }^{2}$, Brendan Keating ${ }^{3}$ and Amein Al-Ali ${ }^{1}$
}

\begin{abstract}
Background: Coronary artery disease (CAD) is a leading cause of morbidity and mortality worldwide. Many genetic and environmental risk factors including atherogenic dyslipidemia contribute towards the development of CAD. Functionally relevant mutations in the dyslipidemia-related genes and enzymes involved in the reverse cholesterol transport system are associated with CAD and contribute to increased susceptibility of myocardial infarction (MI).

Method: Blood samples from 990 angiographically confirmed Saudi CAD patients with at least one event of myocardial infarction were collected between 2012 and 2014. A total of 618 Saudi controls with no history or family history of CAD participated in the study. Four polymorphisms, rs2230806, rs2066715 (ABCA1), rs5882, and rs708272 (CETP), were genotyped using TaqMan Assay.

Results: CETP rs5882 (OR $=1.45, P<0.005)$ and $A B C A 1$ rs2230806 (OR $=1.42, P=0.017)$ polymorphisms were associated with increased risk of CAD. However, rs708272 polymorphism showed protective effect (B1 vs. $B 2: \mathrm{OR}=0.80, P=0.003$ and $\mathrm{B} 2 \mathrm{~B} 2$ vs. $\mathrm{B} 1 \mathrm{~B} 1$ : $\mathrm{OR}=0.68, P=0.012$ ) while the $A B C A 1$ variant $\mathrm{rs} 2066715$ was not associated.

Conclusion: This study is the first to report the association of these polymorphisms with CAD in the population of the Eastern Province of Saudi Arabia. The rs5882 polymorphism (CETP) showed a significant association and therefore could be a promising marker for CAD risk estimation while the rs708272 polymorphism had a protective effect from CAD.
\end{abstract}

Keywords: Gene polymorphism, CAD, CETP, ABCA1, TaqMan Assay

\section{Background}

Coronary artery disease (CAD) is one of the leading causes of morbidity and disability and the most common cause of mortality worldwide equally among men and women. CAD is a disease burden in both high- and lowincome countries [1,2]. A study conducted on 17,232 people from Saudi Arabia revealed that $5.5 \%$ had been diagnosed with CAD, with a higher prevalence in urban populations $(6.2 \%)$ compared to rural populations (4\%) [3]. Platelet aggregation and thrombus formation following the rupture of coronary atherosclerotic plaque is the major cause of myocardial infarction (MI) [4-6].

\footnotetext{
* Correspondence: ccyrus@uod.edu.sa

${ }^{1}$ Institute for Research and Medical Consultation, University of Dammam,

P.O.Box 1982, Dammam 31441, Kingdom of Saudi Arabia

Full list of author information is available at the end of the article
}

Many extrinsic and intrinsic risk factors, including hypertension, dyslipidemia, obesity, smoking, age, lack of exercise, and diabetes, are established risk factors for MI [7]. Atherogenic dyslipidemia is characterized by abnormal levels of triglycerides, low- and high-density lipoprotein (LDL-C and HDL-C) [8-10]. Functionally relevant mutations in the dyslipidemia-related genes and gene encoding enzymes involved in the reverse cholesterol transport system have been reported to be associated with high-density lipoprotein-cholesterol (HDL-C) levels [11-13]. Epidemiological and clinical studies have demonstrated a contradictory association between HDL-C concentrations and cardiovascular risk [10, 14, 15]. The anti-atherogenic effect of HDL-C may act through several mechanisms, such as anti-oxidation of low-density 
lipoprotein-cholesterol (LDL-C) and anti-inflammation and inhibition of vascular endothelial cell apoptosis.

The reverse cholesterol transport system plays a vital role in these processes [16], as it is involved in the transportation of cholesterol from the peripheral tissues to the liver, where cholesterol is secreted into bile. ATP-bindingcassette A1 (ABCA1), apolipoprotein A-1 (ApoA-1), and cholesteryl ester transfer protein (CETP) play important roles in the reverse cholesterol transport system [17]. Certain $A B C A 1$ polymorphisms have been reported to be associated with HDL-C concentrations, which in turn indicate increased cardiovascular risk [18]. The potential atherogenicity of CETP relates to its ability to transfer cholesteryl esters from the anti-atherogenic HDLs to the pro-atherogenic VLDL and LDL proteins. Mutations in the CETP gene give rise to less functional protein, which reduces the transfer of cholesteryl esters, and consequently HDL levels are elevated [19]. ABCA1 and CETP, variants including rs2230806, rs2066715, and rs5882, have been associated with increased HDL-C concentrations and rs708272 with a decreased risk for CAD [20, 21].

The objective of the present study is to evaluate the association of the two $A B C A 1$ polymorphisms, rs2230806 [R219K: c.656G>A (p.Arg219Lys)] and rs2066715 [V825I: c.2473G >A (p.Val825Ile)], and two CETP polymorphisms, rs5882 [V422I: c.1264G>A (p.Val422Ile)] and rs708272 [TaqIB: c.118+279G>A], with the risk of CAD in the population of the Eastern Province of Saudi Arabia.

\section{Results}

Demographical and clinical data of cases and the control group, including age, sex, clinical manifestations, and biochemical parameters, are shown in Table 1. Patients were classified into subgroups based on their hypertension and diabetes status. Hypertension and diabetes were more prevalent in the patient group compared to the control group.

All genotype frequencies of the control group were consistent with Hardy-Weinberg equilibrium. The distribution

Table 1 Demographic and clinical characteristics of study subjects

\begin{tabular}{llll}
\hline Baseline characteristics & Cases $(N=990)$ & Controls $(N=618)$ & $P$ value \\
\hline Age (years) & $58.37 \pm 12.91$ & $54.8 \pm 8.5$ & $<0.0001^{\mathrm{a}}$ \\
Sex M:F & $708: 282$ & $423: 195$ & $0.19^{\mathrm{b}}$ \\
Total cholesterol $(\mathrm{mg} / \mathrm{dl})$ & $170.84 \pm 48.39$ & $171.58 \pm 43.25$ & $0.50^{\mathrm{a}}$ \\
LDL cholesterol $(\mathrm{mg} / \mathrm{dl})$ & $103.82 \pm 40.58$ & $107.25 \pm 35.35$ & $0.005^{\mathrm{a}}$ \\
HDL cholesterol $(\mathrm{mg} / \mathrm{dl})$ & $36.19 \pm 9.88$ & $50.22 \pm 23.89$ & $<0.0001^{\mathrm{a}}$ \\
Clinical Characteristics & & & \\
Hypertension, $n(\%)$ & $688(69.5)$ & $74(12.0)$ & $<0.05^{\mathrm{b}}$ \\
Diabetes, $n(\%)$ & $577(58.3)$ & $87(14.1)$ & $<0.05^{\mathrm{b}}$ \\
\hline
\end{tabular}

atudent's $t$ test

${ }^{\mathrm{b}}$ Chi-square test of analyzed genotype polymorphisms are shown in Table 2. Since all the four SNPs had a G>A transition substitution, the genotypes are denoted with the amino acid change, except for Taq1B alleles, which are designated by $\mathrm{B} 1$ and $\mathrm{B} 2$. The genotype analysis showed overall heterozygous polymorphism predominance in rs2230806 of $A B C A 1$, rs5882, and rs708272 of CETP (Table 2).

The CETP rs708272 polymorphism showed a significantly lower risk for $\mathrm{CAD}(\mathrm{B} 1 \mathrm{~B} 2+\mathrm{B} 2 \mathrm{~B} 2$ vs. B1B1: $\mathrm{OR}=$ $0.68,95 \%$ CI $0.55-0.85, P=0.0006$ and $\mathrm{B} 2 \mathrm{~B} 2$ vs. B1B1: $\mathrm{OR}=0.68,95 \% \mathrm{CI} 0.50-0.92, P=0.012$ ). There was also a significant variation of B1B2 genotypes among patients and controls $(\mathrm{OR}=0.68,95 \%$ CI $0.54-0.86, P=0.001)$. Genotyping for the rs5882 polymorphism in CETP exon14 showed that the frequency of VI genotype was higher in cases than in controls (52.8 vs. $48.0 \%$ ). Our analysis revealed that CETP rs5882 polymorphism is associated with an increased risk of CAD in our Saudi population study dataset (VI+II vs. VV: $\mathrm{OR}=1.42,95 \%$ CI 1.11-1.82, $P=0.005$; II vs. VV: OR $=1.37,95 \%$ CI $1.02-1.82, P=$ 0.031 ). Allele frequency analysis of the $\mathrm{B} 2$ allele of rs708272 of CETP (OR $=0.80,95 \%$ CI 0.69-0.92, $P=0.003)$ and the $\mathrm{K}$ allele of rs2230806 of $A B C A 1$ (OR $=1.17,95 \%$ CI $1.01-$ $1.35, P=0.029)$ showed a significant difference between the two tested groups (Table 2). The mutant KK genotype of rs2230806 of $A B C A 1$ is found to be associated with an increased risk of CAD (RR vs. KK: OR $=1.42,95 \%$ CI 1.06$1.91, P=0.017)$. There were no significant differences in allele and genotype frequencies of rs2066715 polymorphisms in $A B C A 1$ between the patient and control groups. The power of the study observed was $100 \%$ for protective effect at odds ratio of 0.5 and $94.9 \%$ at 0.7 for TaqIB, and for the other three SNPs (R219K, V825I, and I405V), the results ranged from 45.48 to $96.9 \%$ for an odds ratio of 1.2-1.5.

A joint analysis of two SNPs of both $A B C A 1$ and CETP is shown in Table 3 . All the combinations of the CETP variants exhibited no association, except $\mathrm{B} 1 \mathrm{~B} 1+\mathrm{VI}$ $(\mathrm{OR}=1.7,95 \%$ CI 1.0-2.9, $P=0.048)$. On the other hand, for $A B C A 1, \mathrm{RK}+\mathrm{VV}$ and $\mathrm{RK}+\mathrm{VI}$ lacked an association (Table 3). A sex-based analysis revealed a higher frequency of B1B1 genotype in men and women with CAD compared to their respective controls (Table 4). There was no statistical significance in the distribution of $A B C A 1$ genotypes in the female cohort whereas in the male cohort genotypes $\mathrm{KK}(\mathrm{OR}=1.8, P=0.001)$ of rs2230806 and VI $(\mathrm{OR}=2.17, P=0.041)$ of rs2066715 showed a significantly higher risk for CAD. In the CETP, rs5882 polymorphisms II (OR $=1.98,95 \%$ CI $1.38-2.85$, $P=0.0002)$ revealed a significantly higher risk for $C A D$ in the male cohort. In the male and female cohorts, rs708272 B1B2 and B2B2 genotypes showed a protective effect for CAD, respectively. The rs2230806 and rs2066715 of $A B C A 1$ did not show any significant 
Table 2 Association between ABCA1 and CETP genotypes and alleles with CAD

\begin{tabular}{|c|c|c|c|c|c|}
\hline SNP & Genotype/alleles & Cases $(N=990)$ & Control $(N=618)$ & OR $(95 \%$ Cl) & $P$ value \\
\hline \multirow[t]{6}{*}{ rs2230806 (R219K) } & $\mathrm{RR}$ & 291 & 195 & Ref & \\
\hline & RK & 473 & 317 & $0.9(0.79-1.25)$ & 0.999 \\
\hline & KK & 226 & 106 & $1.42(1.06-1.91)$ & 0.017 \\
\hline & $\mathrm{RK}+\mathrm{KK}$ & 699 & 423 & $1.10(0.89-1.37)$ & 0.359 \\
\hline & $\mathrm{R}(\mathrm{G})$ & 1055 & 707 & Ref & \\
\hline & $K(A)$ & 925 & 529 & $1.17(1.01-1.35)$ & 0.029 \\
\hline \multirow[t]{6}{*}{ rs2066715 (V825I) } & W & 945 & 597 & Ref & \\
\hline & $\mathrm{VI}$ & 45 & 21 & $1.35(0.79-2.29)$ & 0.26 \\
\hline & $\|$ & 0 & 0 & - & - \\
\hline & $\mathrm{VI}+\mathrm{II}$ & 45 & 21 & $1.35(0.79-2.29)$ & 0.26 \\
\hline & $V(G)$ & 1935 & 1215 & Ref & \\
\hline & $I(A)$ & 45 & 21 & $1.34(0.79-2.26)$ & 0.27 \\
\hline \multirow[t]{6}{*}{ rs5882 (V422I) } & W & 178 & 147 & Ref & \\
\hline & $\mathrm{Vl}$ & 523 & 297 & $1.45(1.12-1.88)$ & 0.005 \\
\hline & $\|$ & 289 & 174 & $1.37(1.02-1.82)$ & 0.031 \\
\hline & $\mathrm{VI}+\| \mathrm{I}$ & 812 & 471 & $1.42(1.11-1.82)$ & 0.005 \\
\hline & $V(G)$ & 879 & 591 & Ref & \\
\hline & $I(A)$ & 1101 & 645 & $1.14(0.99-1.32)$ & 0.058 \\
\hline \multirow[t]{6}{*}{ rs708272 (TaqlB) } & B1B1 & 376 & 183 & Ref & \\
\hline & B1B2 & 454 & 321 & $0.68(0.54-0.86)$ & 0.001 \\
\hline & B2B2 & 160 & 114 & $0.68(0.50-0.92)$ & 0.012 \\
\hline & $\mathrm{B} 1 \mathrm{~B} 2+\mathrm{B} 2 \mathrm{~B} 2$ & 614 & 435 & $0.68(0.55-0.85)$ & 0.0006 \\
\hline & $\mathrm{B} 1(\mathrm{G})$ & 1206 & 687 & Ref & \\
\hline & $\mathrm{B} 2(\mathrm{~A})$ & 774 & 549 & $0.80(0.69-0.92)$ & 0.003 \\
\hline
\end{tabular}

Table 3 Association between joint analysis of two SNPs of ABCA1 gene and CETP gene with CAD

\begin{tabular}{|c|c|c|c|c|c|c|}
\hline Gene & Genotype & Cases $(N=990)$ & Controls $(N=618)$ & OR & $95 \% \mathrm{Cl}$ & $P$ value \\
\hline \multirow[t]{5}{*}{$A B C A 1$} & $\mathrm{RR}+\mathrm{W}$ & 256 & 186 & Ref & & \\
\hline & $\mathrm{RR}+\mathrm{VI}$ & 35 & 9 & 2.82 & $1.32-6.02$ & 0.007 \\
\hline & $\mathrm{RK}+\mathrm{W}$ & 463 & 305 & 1.10 & $0.86-1.39$ & 0.419 \\
\hline & $\mathrm{RK}+\mathrm{VI}$ & 10 & 12 & 0.60 & $0.25-1.43$ & 0.252 \\
\hline & $\mathrm{KK}+\mathrm{W}$ & 226 & 106 & 1.54 & $1.14-2.08$ & 0.004 \\
\hline \multirow[t]{9}{*}{ CETP } & $\mathrm{B} 1 \mathrm{~B} 1+\mathrm{W}$ & 46 & 31 & Ref & & \\
\hline & $\mathrm{B} 1 \mathrm{~B} 1+\mathrm{VI}$ & 180 & 71 & 1.70 & $1.0-2.90$ & 0.048 \\
\hline & $\mathrm{B} 1 \mathrm{~B} 1+\mathrm{II}$ & 150 & 81 & 1.24 & $0.73-2.11$ & 0.41 \\
\hline & $\mathrm{B} 1 \mathrm{~B} 2+\mathrm{W}$ & 77 & 71 & 0.73 & $0.41-1.27$ & 0.27 \\
\hline & $\mathrm{B} 1 \mathrm{~B} 2+\mathrm{VI}$ & 269 & 175 & 1.03 & $0.63--1.69$ & 0.888 \\
\hline & $\mathrm{B} 1 \mathrm{~B} 2+\mathrm{II}$ & 108 & 75 & 0.97 & $0.56-1.66$ & 0.913 \\
\hline & $\mathrm{B} 2 \mathrm{~B} 2+\mathrm{W}$ & 55 & 45 & 0.82 & $0.45-1.50$ & 0.527 \\
\hline & $\mathrm{B} 2 \mathrm{~B} 2+\mathrm{VI}$ & 74 & 51 & 0.97 & $0.54-1.74$ & 0.939 \\
\hline & $\mathrm{B} 2 \mathrm{~B} 2+\mathrm{II}$ & 31 & 18 & 1.16 & $0.55-2.42$ & 0.692 \\
\hline
\end{tabular}


Table 4 Association between ABCA1 and CETP genotypes and CAD in male and female cohorts

\begin{tabular}{|c|c|c|c|c|c|c|c|c|c|}
\hline \multicolumn{2}{|c|}{ Genotype } & \multicolumn{4}{|l|}{ Male cohort } & \multicolumn{4}{|l|}{ Female cohort } \\
\hline & & Cases $(n=708)$ & Control $(n=423)$ & OR (95 \% Cl) & $P$ value & Cases $(n=282)$ & Control $(n=195)$ & OR $(95 \%$ Cl) & $P$ value \\
\hline \multirow[t]{3}{*}{ R219K } & $\mathrm{RR}$ & 212 & 137 & Ref & & 79 & 58 & Ref & \\
\hline & RK & 337 & 229 & $0.95(0.72-1.24)$ & 0.718 & 136 & 88 & $1.13(0.73-1.74)$ & 0.566 \\
\hline & KK & 159 & 57 & $1.80(1.24-2.61)$ & 0.001 & 67 & 49 & $1.0(0.60-1.65)$ & 0.987 \\
\hline \multirow[t]{2}{*}{ V825I } & W & 676 & 414 & Ref & & 269 & 183 & Ref & \\
\hline & $\mathrm{VI}$ & 32 & 9 & $2.17(1.02-4.60)$ & 0.041 & 13 & 12 & $0.73(0.32-1.65)$ & 0.458 \\
\hline \multirow[t]{3}{*}{ V422I } & W & 123 & 103 & Ref & & 55 & 44 & Ref & \\
\hline & $\mathrm{VI}$ & 376 & 232 & $1.35(0.99-1.84)$ & 0.052 & 147 & 65 & $1.80(1.10-2.96)$ & 0.018 \\
\hline & $\|$ & 209 & 88 & $1.98(1.38-2.85)$ & 0.0002 & 80 & 86 & $0.74(0.45-1.22)$ & 0.246 \\
\hline \multirow[t]{3}{*}{ TaqlB } & B1B1 & 261 & 124 & Ref & & 115 & 59 & Ref & \\
\hline & B1B2 & 340 & 247 & $0.65(0.49-0.85)$ & 0.002 & 114 & 74 & $0.79(0.51-1.21)$ & 0.282 \\
\hline & B2B2 & 107 & 52 & $0.97(0.65-1.45)$ & 0.91 & 53 & 62 & $0.43(0.27-0.71)$ & 0.0008 \\
\hline
\end{tabular}

association with an increased risk for CAD in the female cohort compared to the control subjects.

\section{Discussion}

$\mathrm{CAD}$ is a multifactorial disease mediated through a complex association of environmental and genetic factors with ethnicity demonstrated to be an important determinant of disease variability. The strength of the current study is the selection of CAD patients from similar ethnic backgrounds. In the present study, two common genetic variations in each $A B C A 1$ and CETP gene were studied with reference to their effect on CAD. We tested four SNPs, namely rs2230806 (R219K), rs2066715 (V825I), rs5882 (V422I), and rs708272 (TaqIB) for their association with CAD. CETP, located on chromosome $16 q 21$, plays a crucial role in lipid metabolism, and numerous SNPs in this gene have been reported to alter the plasma HDL-C levels and function of CETP $[22,23]$. Among the CETP SNPs, rs708272 is the one that is most studied. Therefore, we investigated the association of two SNPs of this gene and their risk for CAD in the Saudi population.

Our overall results showed that the heterozygous and mutant of rs708272 polymorphism may confer protection against $\mathrm{CAD}$ (B1B2: $\mathrm{OR}=0.68, P=0.001$; $\mathrm{B} 2 \mathrm{~B} 2$ : $\mathrm{OR}=$ $0.68, P=0.012)$ while those of rs5882 increased the risk of CAD (VI: $\mathrm{OR}=1.45, P=0.005$; II: $\mathrm{OR}=1.37, P=0.031$ ). Earlier studies had suggested that the CETP variant rs5882 causes low CETP and is associated with higher HDL and possibly with increased CAD among hypertriglyceridemia men [24, 25]. In contrast, in many recent studies, the rs5882 polymorphism lacked any association with CAD [26-29]. ABCA1 encodes an important protein that facilitates the formation of HDL-C and regulates the efflux of lipids from peripheral cells into lipid-poor ApoA1 particles, stimulating reverse cholesterol transport. [30] The association between the $A B C A 1$ gene polymorphisms and CAD has been the focus for many studies [31-33]. The rs2230806 is the most common polymorphism of $A B C A 1$; the possible role of rs2230806 in cardiovascular diseases is still debatable as numerous studies have reported divergent results $[34,35]$. The results of our study revealed that the $\mathrm{K}$ variant of $\operatorname{rs} 2230806(P=0.029)$ is associated with CAD, which is in line with Zargar et al. [36]. The rs2066715 of $A B C A 1$ is not associated with an increased risk of $C A D$. The frequency of the rare allele of rs2066715 (0.02) showed a unique distribution compared to the 1000 genome database (0.113) and Han Chinese (0.44) population [37]. The $\mathrm{K}$ allele frequency of rs2230806 (R219K) polymorphism in our study was 0.47 compared to $0.28-0.73$ in earlier studies [18, 38, 39]. The $\mathrm{K}$ allele frequency reported in our study is in line with other reports, namely a European ancestry study which reported a $\mathrm{K}$ allele frequency of $0.26-0.46$ and a study on Dutch men with proven $\mathrm{CAD}$, which reported a frequency of $0.46[40,41]$.

An analysis of the effect of a combination of $A B C A 1$ genotypes on $\mathrm{CAD}$ showed that the $\mathrm{RR}+\mathrm{VI}$ genotype was significantly associated with a high risk of CAD $(\mathrm{OR}=2.82$, $95 \%$ CI 1.32-6.02, $P=0.007)$. However, an analysis of the effect of the combination of CETP genotypes on CAD showed no significant pattern. All other combinations lacked a significant association with an OR ranging from 0.73 to 1.24 , except for $\mathrm{B} 1 \mathrm{~B} 1+\mathrm{VI}(\mathrm{OR}=1.7$, $P=0.048)$. The prevalence of CAD was higher in males (M:F 708:282) than in females in the present study. Homozygous mutant and heterozygous of rs5882 in men and women were strongly associated with an increased risk of $C A D$, while the other two $A B C A 1$ polymorphisms showed no significant association with CAD (OR $=0.73-$ 1.13) in the female cohort. Also, heterozygousity of rs708272 alleles was strongly associated with an increased risk of $\mathrm{CAD}(\mathrm{OR}=0.65, P=0.002)$, a homozygous carriage of rs5882, the rarer variants II causing amino acid 
substitutions showed a strong association with an increased risk of CAD $(\mathrm{OR}=1.98, P=0.0002)$ among the male cohort. The CETP rs5882 polymorphism was found to be associated with an increased risk for CAD in the overall study population and also in the male and female cohorts, which is in contrast to a recent study which reported that this polymorphism is associated with a decreased risk of CAD and MI [42].

Studies conducted in other populations have correlated the genotypes of $A B C A 1$ and $C E T P$ and risk of CHD with respect to its effect on HDL-C. A study of 119 patients in Korea showed that the B1B1 genotype of the CETP Taq1B polymorphism was associated with low HDL-C levels in females and non-smoking males and may be an independent genetic risk factor for CAD [43]. In the present study, the $\mathrm{B} 2$ allele of the CETP rs708272 polymorphism was associated with a reduced risk of CAD mediated by elevated HDL-C concentrations. However, Borggreve et al. in their prospective population-based study (PREVENT study) on 8141 Caucasians demonstrated that the B2 and I alleles of the rs708272 (TaqIB) and rs5882 (V422I) of the CETP gene were not associated with a decreased risk for CAD, despite their HDL-C-raising effect suggesting that the risk may be independent of the gene's influence on HDL-C levels [44]. Thus, the association of polymorphic CETP genotypes with a decreased cardiovascular risk seems to be independent of their effect on HDL-C levels [45-47].

\section{Conclusion}

This study is the first to report the association of these polymorphisms with the development of CAD in a Saudi population. A significant association of CETP rs5882 and $A B C A 1$ rs2230806 polymorphism with CAD was observed marking these polymorphisms as risk factors. The rs708272 showed a protective effect for CAD, and rs2066715 of $A B C A 1$ gene lacked any association with $\mathrm{CAD}$, whereas the joint effect of the $A B C A 1$ gene (RR $+\mathrm{VI}$ and $\mathrm{KK}+\mathrm{VV}$ ) conferred a higher risk for CAD. A sex difference subsists with a higher prevalence of CAD among males (M:F 708:282). Female heterozygous and male homozygous for the rs5882 were shown to have an increased risk of CAD.

\section{Methods}

\section{Study population}

Patients reporting to the cardiac clinic at King Fahd Hospital of the University, Al-Khobar, and other major hospitals in the Eastern Province of Saudi Arabia were screened, and angiographically confirmed CAD cases with at least one event of MI $(N=990)$ were enrolled in this study. A total of 618 age-matched normal Saudi controls with no history or family history of CAD were recruited from the blood banks of the same hospitals. This study was approved by the Ethical Committee of the University of Dammam. Signed written informed consent was obtained from all participants.

\section{Genotyping}

Blood samples $(5 \mathrm{ml})$ were obtained from 1608 subjects in EDTA-coated tubes and DNA was extracted using QIAamp DNA isolation kit (Qiagen, Germany) as per the manufacturer's instructions. Allele-Specific TaqMan PCR procedures were used to detect the genetic variants rs2230806, rs2066715, rs708272, and rs5882.

\section{Statistical analysis}

The difference between cases and controls was evaluated using $t$ test for continuous variables and Chi-square test for discrete variables. Allele frequencies were estimated by direct counting of the test allele divided by the total number of alleles. To assess the risk for CAD, odds ratio was determined by univariate analysis. All statistical analyses were performed using SPSS software (version19). The power of the study was calculated using online software sampsize.sourceforge.net.

\section{Competing interests \\ The authors declare that they have no competing interests.}

\section{Authors' contributions}

CC, CV, and SC designed the study, performed the assay, and drafted the manuscript. AAS and MA provided the CAD patient samples, AAN and FAM provided the age- and sex-matched controls. MSA and RAA collected all medical data of the individual participant from the hospital records. CV performed the statistical analyses. BK was involved in drafting the manuscript for important intellectual content. AAA and FAM provided critical review of the manuscript. All authors made significant intellectual contributions and have read and reviewed the manuscript. All authors read and approved the final manuscript.

\section{Acknowledgements}

The authors acknowledge the King Abdulaziz City for Science and Technology (KACST) for funding the for the current research grant (LGP 32-44). We thank Mr. Geoffrey James Tam Moro and Mr. Florentino Jr Mata for the technical support.

\section{Author details}

${ }^{1}$ Institute for Research and Medical Consultation, University of Dammam, P.O.Box 1982, Dammam 31441, Kingdom of Saudi Arabia. ${ }^{2}$ King Fahd Hospital of the University, University of Dammam, P.O.Box 4001, Al-Khobar 31952, Kingdom of Saudi Arabia. ${ }^{3}$ Department of Pediatrics, Perelman School of Medicine, University of Pennsylvania, Philadelphia, PA, USA.

Received: 3 November 2015 Accepted: 26 February 2016 Published online: 02 March 2016

References

1. Kubo M, Kiyohara Y, Kato I, et al. Trends in the incidence, mortality, and survival rate of cardiovascular disease in a Japanese community: the Hisayama study. Stroke. 2003;34(10):2349-54.

2. Gaziano TA, Bitton A, Anand S, et al. Growing epidemic of coronary heart disease in low-and middle-income countries. Curr Probl Cardiol. 2010;35(2): 72-115.

3. Al-Nozha MM, Arafah MR, Al-Mazrou YY, et al. Coronary artery disease in Saudi Arabia. Saudi Med J. 2004;25(9):1165-71.

4. Yamada $Y$, Izawa $H$, Ichihara $S$, et al. Prediction of the risk of myocardial infarction from polymorphisms in candidate genes. N Engl J Med. 2002; 347(24):1916-23. 
5. Licastro F, Chiappelli M, Porcellini E, et al. Gene-gene and gene-clinical factors interaction in acute myocardial infarction: a new detailed risk chart. Curr Pharm Des. 2010;16(7):783-8.

6. Ohki T, Itabashi Y, Kohno T, et al. Detection of periodontal bacteria in thrombi of patients with acute myocardial infarction by polymerase chain reaction. Am Heart J. 2012;163(2):164-7.

7. Romero-Corral A, Somers VK, Sierra-Johnson J, et al. Normal weight obesity: a risk factor for cardio metabolic dysregulation and cardiovascular mortality. Eur Heart J. 2010;31(6):737-46.

8. Vinueza R, Boissonnet CP, Acevedo M, et al. Dyslipidemia in seven Latin American cities: CARMELA study. Prev Med. 2010;50(3):106-11.

9. Pöss J, Custodis F, Werner C, et al. Cardiovascular disease and dyslipidemia: beyond LDL. Curr Pharm Des. 2011;17(9):861-70.

10. Voight BF, Peloso GM, Orho-Melander M, et al. Plasma HDL cholesterol and risk of myocardial infarction: a Mendelian randomization study. Lancet. 2012; 380(9841):572-80.

11. Rejeb J, Omezzine A, Rebhi L, et al. Association of the cholesteryl ester transferprotein Taq1 B2B2 genotype with higher high-density lipoproteincholesterol concentrations and lower risk of coronary artery disease in a Tunisian population. Arch Cardiovasc Dis. 2008;101(10):629-36.

12. van Acker BA, Botma GJ, Zwinderman AH, et al. High HDL cholesterol does not protect against coronary artery disease when associated with combined cholesteryl ester transfer protein and hepatic lipase gene variants. Atherosclerosis. 2008;200(1):161-7.

13. Hiura Y, Shen CS, Kokubo Y, et al. Identification of genetic markers associated with high-density lipoprotein-cholesterol by genome-wide screening in a Japanese population: the Suita study. Circ J. 2009;73(6):1119-26.

14. Holzmann MJ, Jungner I, Walldius G, et al. Dyslipidemia is a strong predictor of myocardial infarction in subjects with chronic kidney disease. Ann Med. 2012;44(3):262-70

15. Holmes MV, Asselbergs FW, Palmer TM, et al. Mendelian randomization of blood lipids for coronary heart disease. Eur Heart J. 2015;36(9):539-50.

16. Tsompanidi EM, Brinkmeier MS, Fotiadou EH, et al. HDL biogenesis and functions: role of $\mathrm{HDL}$ quality and quantity in atherosclerosis. Atherosclerosis. 2010;208:3-9.

17. Nakamura A, Niimura $H$, Kuwabara $K$, et al. Gene-gene combination effect and interactions among $A B C A 1, A P O A 1, S R-B 1$, and CETP polymorphisms for serum high-density lipoprotein-cholesterol in the Japanese population. PLoS One. 2013;8(12):e82046.

18. Porchay I, Péan F, Bellili N, et al. ABCA1 single nucleotide polymorphisms on high-density lipoprotein-cholesterol and overweight: the D.E.S.IR.E study. Obesity. 2006;14(11):1874-9.

19. Barter PJ, Brewer Jr HB, Chapman MJ, et al. Cholesteryl ester transfer protein, a novel target for raising HDL and inhibiting atherosclerosis. Arterioscler Thromb Vasc Biol. 2003;23(2):160-7.

20. Brousseau ME, O'Connor Jr JJ, Ordovas JM, et al. Cholesteryl ester transfer protein TaqIB2B2 genotype is associated with higher HDL cholesterol level and lower risk of coronary heart disease endpoints in men with HDL deficiency: veterans affairs $\mathrm{HDL}$ cholesterol intervention trial. Arterioscler Thromb Vasc Biol. 2002;22(7):1148-54.

21. Boekholdt SM, Kuivenhoven JA, Hovingh GK, et al. CETP gene variation: relation to lipid parameters and cardiovascular risk. Curr Opin Lipidol. 2004;15:393-8.

22. Thompson A, Di Angelantonio E, Sarwar N, et al. Association of cholesteryl ester transfer protein genotypes with CETP mass and activity, lipid levels, and coronary risk. JAMA. 2008;299(23):2777-88.

23. Ridker PM, Paré G, Parker AN, et al. Polymorphism in the CETP gene region, HDL cholesterol, and risk of future myocardial infarction: genome wide analysis among 18245 initially healthy women from the Women's genome health study. Circ Cardiovasc Genet. 2009;2:26-33.

24. Bruce C, Sharp DS, Tall AR. Relationship of HDL and coronary heart disease to a common amino acid polymorphism in the cholesteryl ester transfer protein in men with and without hypertriglyceridemia. J Lipid Res. 1998;39:1071-8.

25. Gudnason V, Kakko S, Nicaud V, et al. Cholesteryl ester transfer protein gene effect on CETP activity and plasma high-density lipoprotein in European populations. The EARS Group. Eur J Clin Invest. 1999;29(2):116-28.

26. Padmaja N, Ravindra KM, Soya SS, et al. Common variants of Cholesteryl ester transfer protein gene and their association with lipid parameters in healthy volunteers of Tamilian population. Clin Chim Acta. 2007:375:140-6

27. Parra ES, Panzoldo NB, Kaplan D, et al. The $1405 \mathrm{~V}$ and Taq1B polymorphisms of the CETP gene differentially affect sub- clinical carotid atherosclerosis. Lipids Health Dis. 2012;11:130.
28. Wang J, Wang $\sqcup$, Zhong $Y$, et al. CETP gene polymorphisms and risk of coronary atherosclerosis in a Chinese population. Lipids Health Dis. 2013;12:176.

29. Wang $Q$, Zhou $S B$, Wang $L$, et al. Seven functional polymorphisms in the CETP gene and myocardial infarction risk: a meta-analysis and metaregression. PLoS One. 2014;9(2):e88118.

30. Hayden MR, Clee SM, Brooks-Wilson A, et al. Cholesterol efflux regulatory protein, Tangier disease and familial high-density lipoprotein deficiency. Curr Opin Lipidol. 2000;11:117-22.

31. Abd El-Aziz TA, Mohamed RH, Hagrass HA. Increased risk of premature coronary artery disease in Egyptians with ABCA1 (R219K), CETP (TaqIB), and LCAT (4886C/T) genes polymorphism. J Clin Lipidol. 2014;8(4):381-9.

32. Marvaki A, Kolovou V, Katsiki N, et al. Impact of 3 common ABCA1 gene polymorphisms on optimal vs non-optimal lipid profile in Greek young nurses. Open Cardiovasc Med J. 2014;8:83-7.

33. Liu N, Hou M, Ren W, et al. The R219K polymorphism on ATP-binding cassette transporter $\mathrm{A} 1$ gene is associated with coronary heart disease risk in Asia population: evidence from a meta-analysis. Cell Biochem Biophys. 2015;71(1):49-55.

34. Hou R, Zhu X, Pan X, et al. ATP-binding cassette transporter A1 R219K polymorphism and ischemic stroke risk in the Chinese population: a meta-analysis. Neurological Sci. 2014;336(1):57-61.

35. Yin YW, Li JC, Gao D, et al. Influence of ATP-binding cassette transporter R219K and M883l polymorphisms on development of atherosclerosis: a meta-analysis of 58 studies. PLoS One. 2014;9(1):e86480.

36. Zargar S, Wakil S, Mobeirek AF, et al. Involvement of ATP-binding cassette, subfamily A polymorphism with susceptibility to coronary artery disease. Biomed Rep. 2013;1(6):883-8.

37. Cao XL, Yin RX, Wu DF, et al. Genetic variant of V825I in the ATP-binding cassette transporter A1 gene and serum lipid levels in the Guangxi Bai Ku Yao and Han populations. Lipids Health Dis. 2011;10:14.

38. Pasdar A, Yadegarfar G, Cumming A, et al. The effect of ABCA1 gene polymorphisms on ischaemic stroke and relationship with lipid profile. BMC Med Genet. 2007;8:30.

39. Kolovou V, Kolovou G, Marvaki A, et al. ATP-binding cassette transporter A1 gene polymorphisms and serum lipid levels in young Greek nurses. Lipids Health Dis. 2011;10:56.

40. Koren-Morag N, Tanne D, Graff E, et al. Low and high density lipoprotein cholesterol and ischemic cerebrovascular disease: the bezafibrate infarction prevention registry. Arch Intern Med. 2002;162:993-9.

41. Clee SM, Zwinderman AH, Engert JC, et al. Common genetic variation in ABCA1 is associated with altered lipoprotein levels and a modified risk for coronary artery disease. Circulation. 2001;103(9):1198-205.

42. Isaacs A, Sayed-Tabatabaei FA, Hofman A, et al. The cholesteryl ester transfer protein $1405 \mathrm{~V}$ polymorphism is associated with increased high-density lipoprotein levels and decreased risk of myocardial infarction: the Rotterdam Study. Eur J Cardiovasc Prev Rehabil. 2007;14(3):419-21.

43. Park KW, Choi JH, Kim HK, et al. The association of cholesteryl ester transfer protein polymorphism with high-density lipoprotein cholesterol and coronary artery disease in Koreans. Clin Genet. 2003;63(1):31-8.

44. Borggreve $\mathrm{SE}$, Hillege $\mathrm{HL}$, Wolffenbuttel $\mathrm{BH}$, et al. An increased coronary risk is paradoxically associated with common cholesteryl ester transfer protein gene variations that relate to higher HDL cholesterol: a population based study. J Clin Endocrinol Metab. 2006;91(9):3382-8.

45. Freeman DJ, Griffin BA, Holmes AP, et al. Regulation of plasma HDL cholesterol and subfraction distribution by genetic and environmental factors: associations between the TaqIB RFLP in the CETP gene and smoking and obesity. Arterioscler Thromb. 1994;14(3):336-44.

46. Fumeron F, Betoulle $D$, Luc G, et al. Alcohol intake modulates the effect of a polymorphism of the cholesteryl ester transfer protein gene on plasma high density lipoprotein and the risk of myocardial infarction. J Clin Invest. 1995; 96(3):1664-71.

47. Dullaart RP, Hoogenberg K, Riemens SC, et al. Cholesteryl ester transfer protein gene polymorphism is a determinant of $\mathrm{HDL}$ cholesterol and of the lipoprotein response to a lipid-lowering diet in type 1diabetes. Diabetes. 1997:46(12):2082-7. 